\title{
Sistemas de Comunicação Multiusuário Baseados em Transformadas Trigonométricas Digitais
}

\author{
Isabela M. C. de Albuquerque Érica F. Paiva Ricardo M. Campello de Souza \\ Depto de Eletrônica e Sistemas, UFPE, 50670-901, Recife, PE \\ E-mail: isabelamcalbuquerque@gmail.com, erica.fonpa@gmail.com, Ricardo@ufpe.br
}

\section{$\underline{\text { RESUMO }}$}

Transformadas discretas têm sido propostas para construir sistemas de comunicação multiusuário. Em particular, sistemas utilizando as transformadas numéricas de Fourier (TNF) [5] e de Hartley (TNH) [3] foram propostos, mas ambos possuem uma severa limitação no número de usuários que se pode utilizar, já que este valor está relacionado ao número de autovalores distintos da matriz de transformação. Para a TNF existem quatro autovalores distintos e para a TNH existem apenas dois [1]. Neste cenário, este trabalho propõe um sistema de comunicação multisuário baseado na transformada digital do cosseno do tipo $2\left(F F C T_{2}\right)$, cujas matrizes de transformação possuem uma quantidade maior de autovalores distintos.

Definição 1. O conjunto de inteiros Gaussianos sobre $G F(p)$ é o conjunto $G I(p)=\{a+j b, a, b \in$ $G F(p)\}$ em que p é um número primo tal que $j^{2} \equiv-1$ é um resíduo não quadrático sobre $G F(p)$.

$\operatorname{Em~GI}(p)$ são de especial interesse os chamados elementos unimodulares.

Definição 2. Seja $\zeta=a+j b$ um inteiro Gaussiano sobre $G F(p)$. $\zeta$ é dito ser unimodular se $a^{2}+b^{2} \equiv$ $1(\bmod p)$.

Existem dezesseis transformadas trigonométricas digitais, oito do cosseno e oito do seno. Este trabalho considera a transformada digital do cosseno, par, unitária, do tipo 2 [6].

Definição 3. Seja $\zeta$ um inteiro Gaussiano unimodular com ordem multiplicativa $2 N(\bmod p)$ e $p \equiv$ $3(\bmod 4)$. A FFCT $T_{2}$ da sequência $x=\left(x_{i}\right), i=0,1, \ldots, N-1, x_{i} \in G F(p)$, é a sequência $X=$ $\left(X_{k}\right), k=0,1, \ldots, N-1, X_{k} \in G I(p)$, com componentes

$$
X_{k} \triangleq \sum_{i=0}^{N-1}\left(\sqrt{\frac{2}{N}}\right) \beta_{i} \cos _{\zeta}\left(k\left(i+\frac{1}{2}\right)\right),
$$

em que

$\cos _{\zeta}(n)=\frac{\zeta^{n}+\zeta^{-n}}{2}, \beta_{i}=\left\{\begin{array}{l}\sqrt{2^{-1}} ; i=0 \text { ou } N, \\ 1 ; \text { c.c. }\end{array}, i, k=0,1, \ldots, N-1\right.$.

A matriz de transformação da $F F C T_{2}$ é dada por

$$
\left[F F C T_{2}\right]=\sqrt{\frac{2}{N}}\left[\begin{array}{ccc}
\beta_{0} & \ldots & \beta_{1} \cos _{\zeta}\left[(N-1)\left(\frac{3}{2}\right)\right] \\
\vdots & \ddots & \vdots \\
\beta_{0} & \ldots & \beta_{N-1} \cos _{\zeta}\left[(N-1)\left(\frac{2(N-1)+1}{2}\right)\right]
\end{array}\right]
$$

em que, devido ao fator $\sqrt{\frac{2}{N}}, 2$ e $N$ devem ser resíduos quadráticos de $p$ [2].

As autossequências da $F F C T_{2}$ têm fundamental importância na construção do sistema apresentado a seguir. 
Definição 4. Uma sequência $x=\left(x_{i}\right), x_{i} \in G F(p)$, é dita ser uma autossequência da $F F C T_{2}$, com autovalor $\lambda \in G F(p)$, quando $X=\lambda x$.

O sistema de comunicação multiusuário proposto neste trabalho consiste em associar as sequências de dados dos usuários às autossequências linearmente independentes da matriz da transformada utilizada. Essas sequências são transmitidas por um canal aditivo sem ruído e, na recepção, podem ser separadas, dado que não pertencem a um mesmo subespaço vetorial. Para montar um esquema com três usuários, por exemplo, é necessário uma transformada cuja matriz possua pelo menos três autovalores distintos. Escolhendo $\mathrm{p}=23$ e $\zeta=12+j 15$, tem-se a matriz

$$
\left[F F C T_{2}\right]=\left[\begin{array}{ccc}
13 & 15 & 12 \\
13 & 0 & 22 \\
13 & 8 & 12
\end{array}\right],
$$

cujos autovalores são $\lambda_{1}=1, \lambda_{2}=2$ e $\lambda_{3}=12$. Cada usuário transmite suas mensagens por meio das autossequências $x_{1}, x_{2}$ e $x_{3}$, que estão associadas aos autovalores distintos. Quando essas autossequências são enviadas por um canal aditivo, a sequência transmitida é $y=x_{1}+x_{2}+x_{3}$. Aplicando duas vezes a $F F C T_{2}$ a $y$, obtém-se o sistema

$$
\left\{\begin{array}{l}
y=x_{1}+x_{2}+x_{3}, \\
F y=x_{1}+2 x_{2}+12 x_{3}, \\
F^{2} y=x_{1}+4 x_{2}+6 x_{3},
\end{array}\right.
$$

cuja solução leva às autossequências

$$
\left\{\begin{array}{l}
x_{1}=21 F^{2} y+5 F y+21 y, \\
x_{2}=16 F^{2} y+22 F y+8 y, \\
x_{3}=9 F^{2} y+19 F y+18 y .
\end{array}\right.
$$

Explorações numéricas envolvendo a $F F C T_{2}$ de comprimento $N \leq 10000$ [4], sugerem que esta transformada possui $\mathrm{N}$ autovalores distintos, o que a torna atraente para este tipo de aplicação. As autossequências da $F F C T_{2}$ são usadas como assinaturas de usuários e a recuperação das mesmas pode ser feita via algoritmos rápidos para computação da $F F C T_{2}$, permitindo, dessa forma, a implementação do sistema por meio de processadores digitais de sinais (DSPs). O desempenho do sistema em presença de ruído está sendo investigado.

Palavras-chave: Comunicação Multiusuário, Transformada Digital do Cosseno, Corpos Finitos

\section{Referências}

[1] D. T. Birtwistle, The eigenstructure of the number theoretic transforms, Signal Processing, 4 (1982) 287-294.

[2] M. Burton, "Elementary Number Theory", McGraw-Hill, 2010.

[3] R.M. Campello de Souza, H. M. de Oliveira, A. N. kauffman, A. J. A. Paschoal, Trigonometry in finite fields and a new Hartley transform, Proc. of the IEEE Internationl Symposium on Information Theory p. 293, Cambridge, 1998.

[4] G. Cariolaro, T. Erseghe, P. Kraniauskas, The fractional discrete cosine transform, IEEE Trans. on Signal Processing, 50 (2002) 902-911.

[5] H. M. Cavalcanti, R. M. Campello de Souza, Autossequências da transformada numérica de Fourier, XXV Simpósio Brasileiro de Telecomunicações, Recife, 2007.

[6] J. B. Lima, R.M. Campello de Souza, Finite field trigonometric transforms, Applicable Algebra in Engineering, Communication and Computing, 22 (2011) 393-411. 